\title{
Aerodynamic instabilities in transonic centrifugal compressor
}

\author{
N. Buffaz ${ }^{1}$ And I. TrebinjaC ${ }^{2, a}$ \\ 1 TURBOMECA, Groupe SAFRAN, 64511 Bordes, France \\ 2 Laboratoire de Mécanique des Fluides et d'Acoustique, UMR CNRS 5509, Ecole Centrale de Lyon, 36 av. Guy de Collongue, \\ 69134 Ecully Cedex, France
}

Received 18 June 2013, Accepted 18 March 2014

\begin{abstract}
This paper presents the analysis of the instabilities inception in a transonic centrifugal compressor for different rotation speeds. The analysis was conducted from experimental results obtained with unsteady pressure sensors implanted in the inducer, vaneless diffuser and vaned diffuser. Beyond the stability limit the compressor enters into a deep surge without any precursor, whatever the speed. The surge process is initiated in the vaned diffuser by a massive boundary layer separation. For low speeds, together with the surge which remains triggered in the diffuser, aerodynamic instabilities are detected in the inducer. These instabilities can be understood as "tip clearance rotating disturbances" because they are generated at the leading edge of the impeller main blades and move along the tip clearance trajectory.
\end{abstract}

Key words: transonic centrifugal compressor / instabilities / surge / mild stall

Résumé - La présente contribution présente l'analyse du déclenchement des instabilités dans un compresseur centrifuge transsonique pour différentes vitesses de rotation. Cette analyse a été menée à partir de résultats expérimentaux obtenus grâce à des capteurs de pression rapides implantés au carter dans la partie inductrice du rouet, dans le diffuseur lisse et dans le diffuseur aubé. Quelle que soit la vitesse de rotation, la rupture aérodynamique du système de compression résulte d'un pompage de type profond sans précurseur, initié dans le diffuseur par un décollement massif des couches limites. Pour les faibles vitesses de rotation, conjointement au pompage qui reste déclenché dans le diffuseur, des instabilités aérodynamiques sont enregistrées dans l'inducteur du rouet. Ces instabilités tournantes et associées au tourbillon de jeu sont induites par une sur-incidence sur les aubes principales du rouet.

Mots clés : Compresseur centrifuge transsonique / instabilités / pompage / décollement tournant

\section{Introduction}

In compressors as the mass flow is reduced the pressure rise increases up to a given point at which further reduction of mass flow leads more or less rapidly to more or less severe instabilities. The conventional terminology for this point is the "surge point" even if different types of instabilities may occur, mainly depending on the compressor and the system in which it is operating [1-3]. The instabilities are typically classified into two main classes: rotating stall and surge which actually are very different phenomena. Rotating stall corresponds to a flow field which is no longer axisymmetric but has a circumferentially non-uniform pattern rotating along the annulus which then contains regions of stalled flow. Depending on the number and size of the stalled cells, the compressor is affected by either part-span rotating stall or full-span rotating stall. On the contrary, when fully developed the

\footnotetext{
${ }^{a}$ Corresponding author:

Isabelle.Trebinjac@ec-lyon.fr
}

surge process is axisymmetric and is characterized by a variation with time of the overall annulus mass flow. The surge process may be violent (process known as "deep surge" with reversed averaged mass flow) or mild (process known as "mild surge" with the operating points orbiting around the surge point). Actually the classification into two classes is not strictly tight because most of the time rotating stall evolves into surge.

Because instabilities can be catastrophic for performance and even damaging for the machine, predicting the onset of instability is an essential part of the description of the compressor performance map. The instability (rotating stall or surge) may be triggered by Long Length Scale Disturbances (LLSD) or Short Length Scale Disturbances (SLSD); the reference length scale being the blade-to-blade passage $[4,5]$. LLSD come from the system and the associated modal waves rotate below around $50 \%$ of the rotor speed. SLSD come from a local perturbation leading to disturbances which rotate typically between 50 and $80 \%$ of the rotor speed and evolving in 


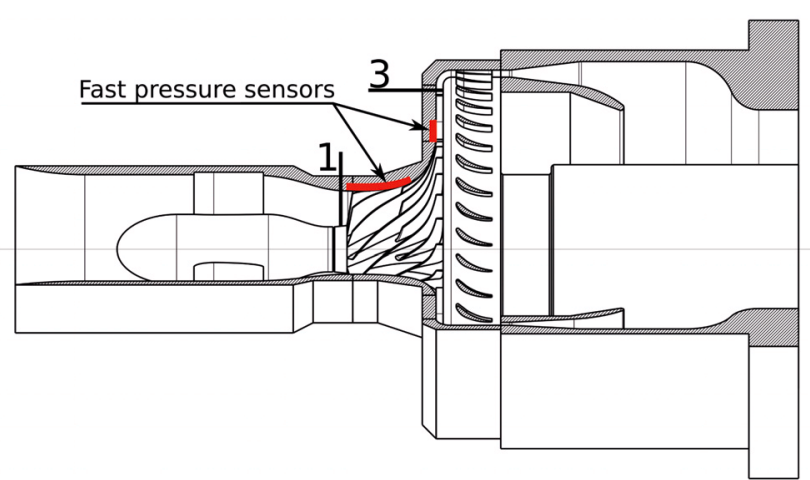

Fig. 1. Meridional view of the compressor.

few revolutions to rotating stall (or surge). Both SLSD and LLSD can be present at the same time and it is also possible to switch between SLSD toward LLSD setting up flow control process [6].

Many works have been dedicated to the description and analysis of the instabilities and their inception but most of them concern axial compressors. In centrifugal compressors, things are a little bit different because centrifugal compressors may satisfactorily operate with stall in the rotor because the largest part of the pressure rise comes from centrifugal effects by change of radii. Consequently surge is the most likely instability which may occur at low mass flow rate even if it is not exclusive $[7,8]$.

The present contribution aims at describing the mechanisms involved in the surge onset in a transonic centrifugal compressor from $60 \%$ to $100 \%$ of the nominal rotation speed. In the first part of the paper, the test case is presented. The aerodynamic instabilities which have been experienced in both the diffuser and inducer are then discussed.

\section{Test case}

The test case is a centrifugal compressor stage, used in a helicopter engine, designed and built by Turbomeca. It is composed of an impeller with axial inflow, a radial vaned diffuser and an axial diffuser as in the real geometry which is therefore axisymmetric. The backswept, unshrouded impeller is composed of $\mathrm{N}_{\mathrm{R}}$ main blades and $\mathrm{N}_{\mathrm{R}}$ splitter blades. The vaned diffuser is composed of $\mathrm{N}_{\mathrm{S}}$ vanes. A meridional view of the stage is given in Figure 1.

The compressor stage is mounted on a $1 \mathrm{MW}$ test rig equipped with 67 steady sensors (temperature, pressure, and vibration measurements) dedicated to monitoring and overall performance measuring. The rotation speed, the flow rate, the pressure and the temperature are measured at $\pm 0.01 \%, \pm 0.5 \%, \pm 0.05 \%$ and $\pm 1 \mathrm{~K}$ respectively.

Unsteady pressure measurements up to $150 \mathrm{kHz}$ were carried out in the inducer of the impeller (between the leading edge of the main blade and the leading edge of the splitter blade), in the vaneless diffuser and in the vaned diffuser with respectively nine, four and eleven sensors (Fig. 2). The sensors placed in the inducer are mounted in a piece of metal set in the shroud over an angular sector of $17^{\circ}$. The sensors placed in the diffuser are also mounted on the shroud. Actually they are distributed over the whole circumference of the machine but, for clarity of presentation, are brought together in Figure 2 as if they were located in a single diffuser passage. The timedependent pressure data were acquired in synchronization with the rotation of the machine. Additional details are given in [9].

\section{Surge line}

Figure 3 gives the static to total pressure ratio of the stage $\left(p_{3} / p_{\mathrm{t} 1}\right)$ as a function of the reduced mass flow at four rotation speeds. The value of $p_{3}$ is the mean value of the static pressures measured on the shroud and hub wall surfaces at the stage outlet (cf. Fig. 1). The reduced mass flow is defined as:

$$
\dot{m}_{\text {red }}=\frac{\dot{m} \sqrt{\gamma r T_{t 1}}}{D_{1 S}^{2} p_{t 1}}
$$

with $T_{t 1}$ and $p_{t 1}$ the stagnation temperature and pressure at Section 1, $D_{1 s}$ the shroud diameter at Section $1, \gamma$ the specific heat ratio and $r$ the gas constant.

Figure 4 gives a sketch of the pressure rise-flow mass curves which are characteristic of a high pressure centrifugal compressor stage (rotor + vaned diffuser) [7,8,10,11]. At high speed, the surge line is typically imposed by the diffuser whereas at low speed, the surge line is imposed by the inducer. In the present case (Fig. 3) the surge line marking the locus of the experimental surge points for the four rotation speeds is perfectly straight which suggests that the surge is triggered by the same component, specifically the diffuser.

\section{Surge inception}

A thorough examination of all the time-dependent pressure signals coming from all the sensors (located in the diffuser and in the inducer) at all rotation speeds led to the definitive conclusion that the surge onset always occurs in the diffuser, which confirms the result stated above. By way of example, Figure 5 gives the pressure signals measured by the sensors I1, I5 and D1 (cf. Fig. 2) during the surge onset. The abscissa is the time reduced by the rotor main blade passing period, the origin being arbitrary. In each signal, the blue curve is the pressure signal filtered below the blade passing frequency and the red line gives the mean pressure value before surge. It can be observed that in both cases $(0.7 \mathrm{Nn}$ on the left and $0.927 \mathrm{Nn}$ on the right) the pre-surge activity is systematically registered at the sensor D1, i.e. in the diffuser.

Actually the onset of surge results from a separation which enlarges on the suction side of the diffuser vanes as the compressor is throttled and which totally blocks the diffuser entry when the surge is fully developed. 

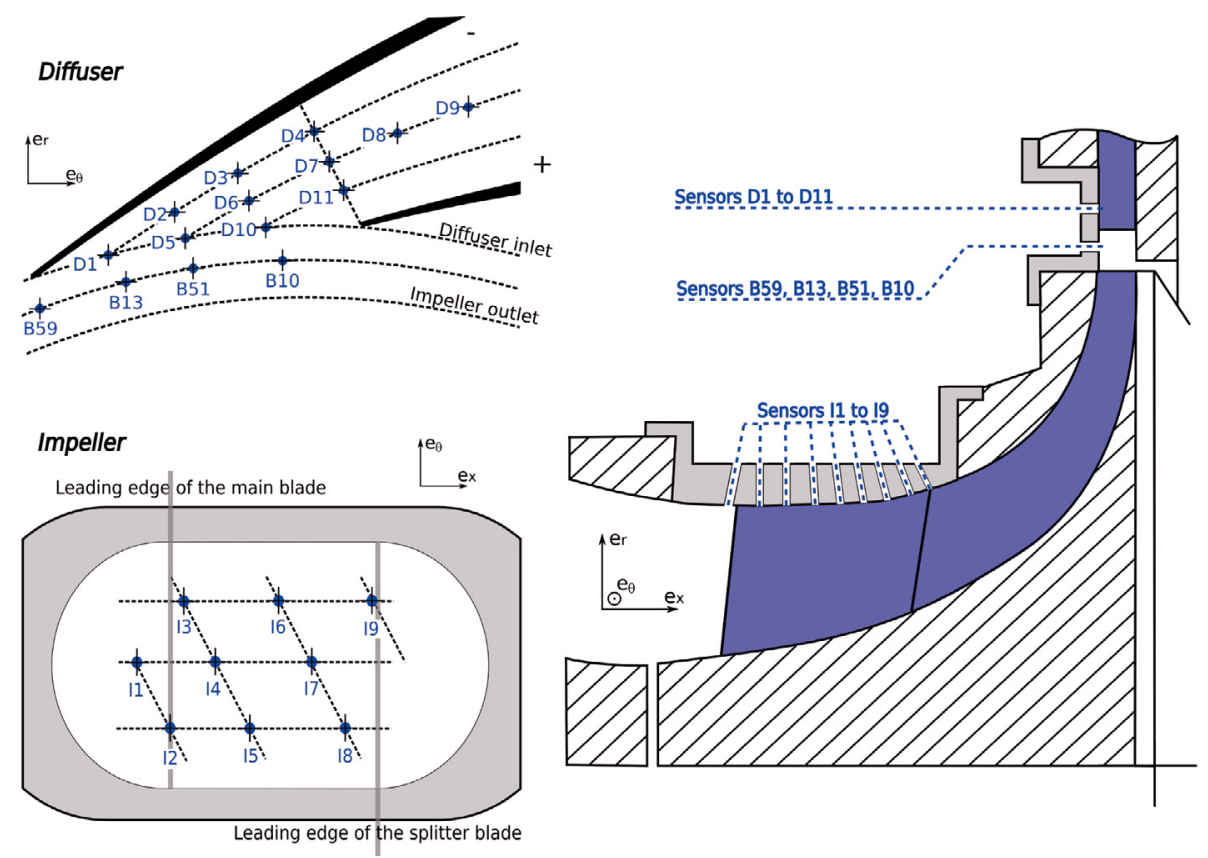

Fig. 2. Location of the fast pressure sensors.

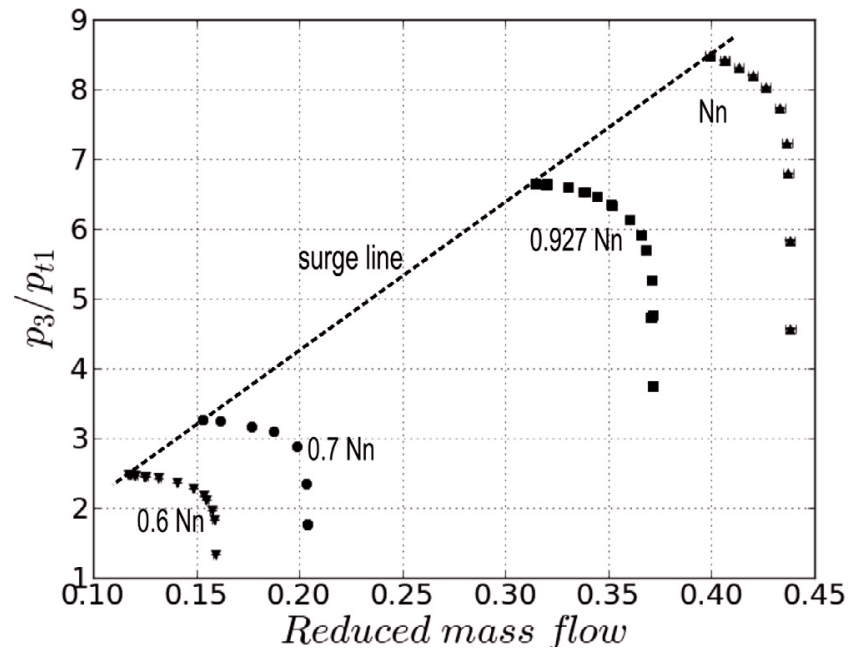

Fig. 3. Static to total pressure ratio of the stage.

Figure 6 shows the maps of the pressure signals filtered below the blade passing frequency (written as $\tilde{p}^{B P F}$ ) in the diffuser passage, at shroud. The pressure level is reduced by a reference value. Each black square represents a pressure sensor. Bringing all the sensors in a single diffuser passage is justified by previous results [12] which have shown that (i) the surge inception is a repeatable process (i.e. not governed by random flow structures); and (ii) the temporal fluctuations are synchronized with the blade passing. The surge inception may thus be considered in the present case as a chorochronic process. It obviously excludes any non-deterministic phenomenon.

Six instants during the surge onset are drawn in Figure 6 . These instants are marked on Figure 7 which gives the pressure signals measured by the sensors B59, D1, D5,

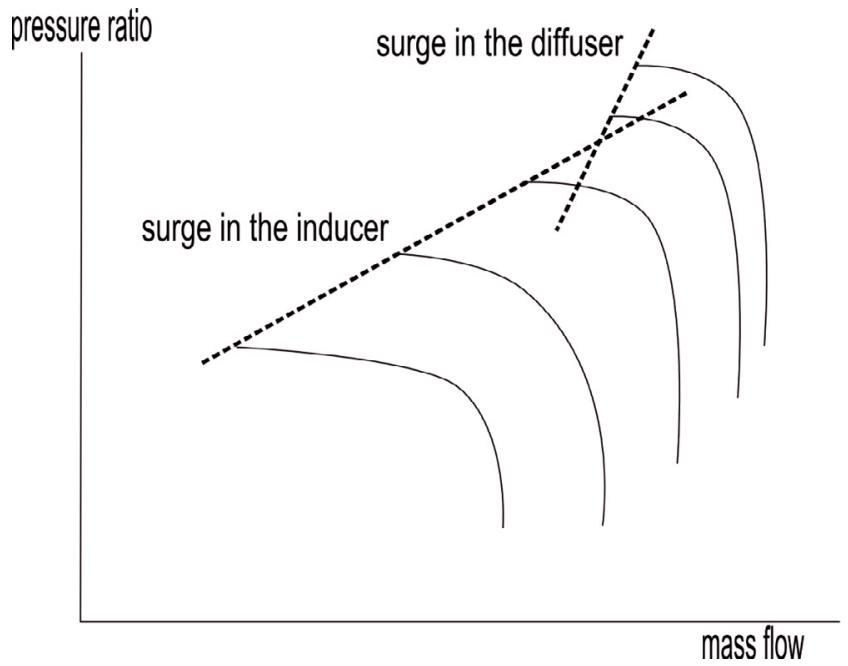

Fig. 4. Schematic pressure rise-mass flow map for a high pressure centrifugal stage.

D10 during the surge onset. The instant (a) is around two revolutions before any sign of "pre-surge activity" is registered (cf. Fig. 7). At this instant (a) the fluctuation level is almost zero. The first pre-surge activity is registered at the sensor D1; it corresponds to the appearance of a separation on the vane suction side due to the interaction between the shock wave and the vane suction side- shroud corner boundary layer. It leads to an increase in pressure (red zone in (b)) which spreads across the diffuser throat whereas the pressure decreases downstream of the throat. It is also interesting to note the slight decrease in pressure at the diffuser entry ((b), (c) and (d)) which comes from the expansion wave resulting from the change in incidence 

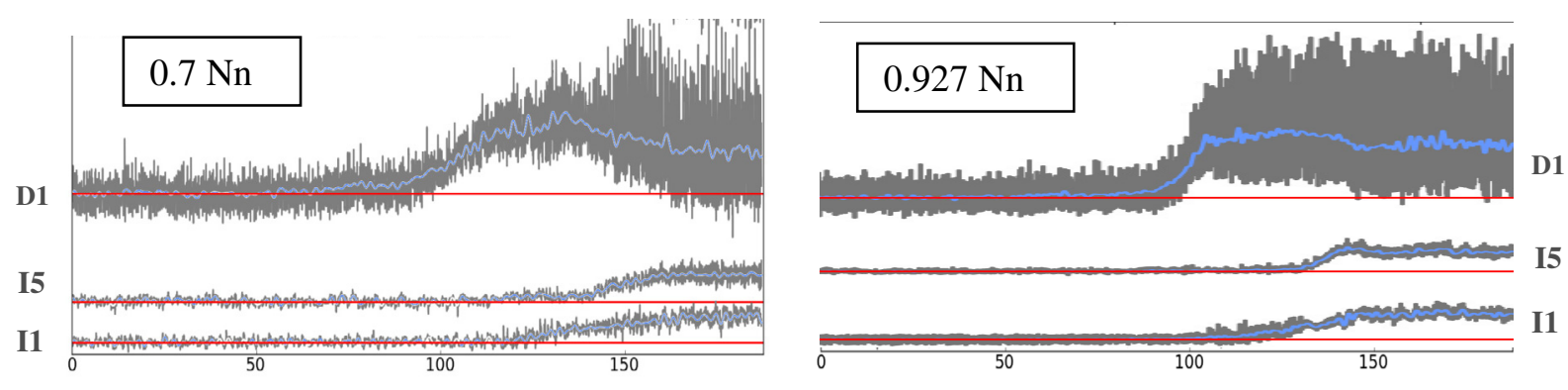

Fig. 5. Pressure signals at sensors I1, I5 and D1.
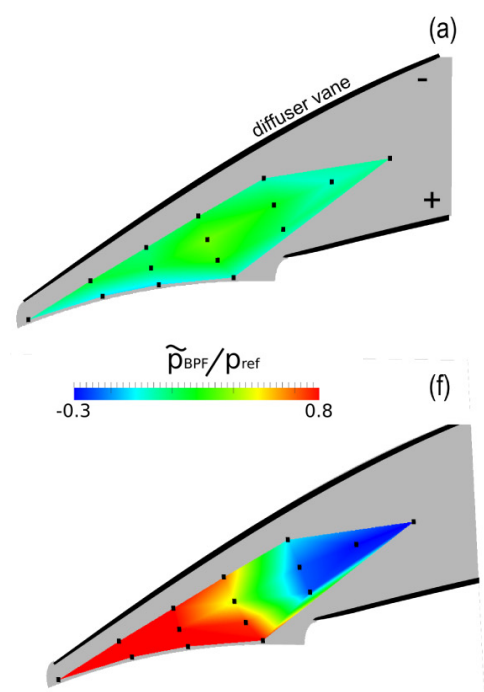

(b)

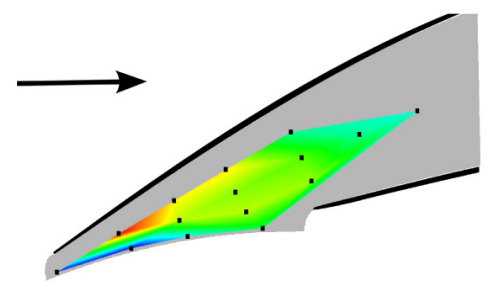

(e)

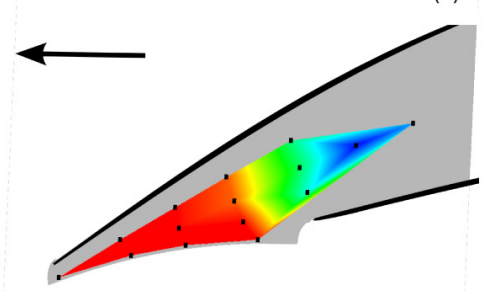

(c)

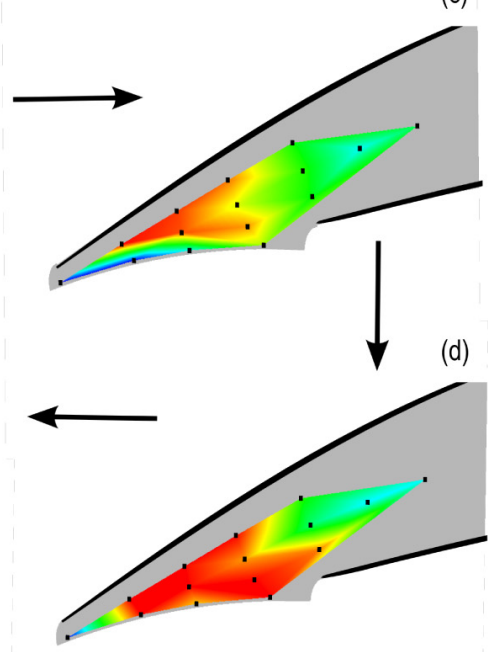

Fig. 6. Pressure filtered below the blade passing frequency during surge inception at $0.927 \mathrm{Nn}$.

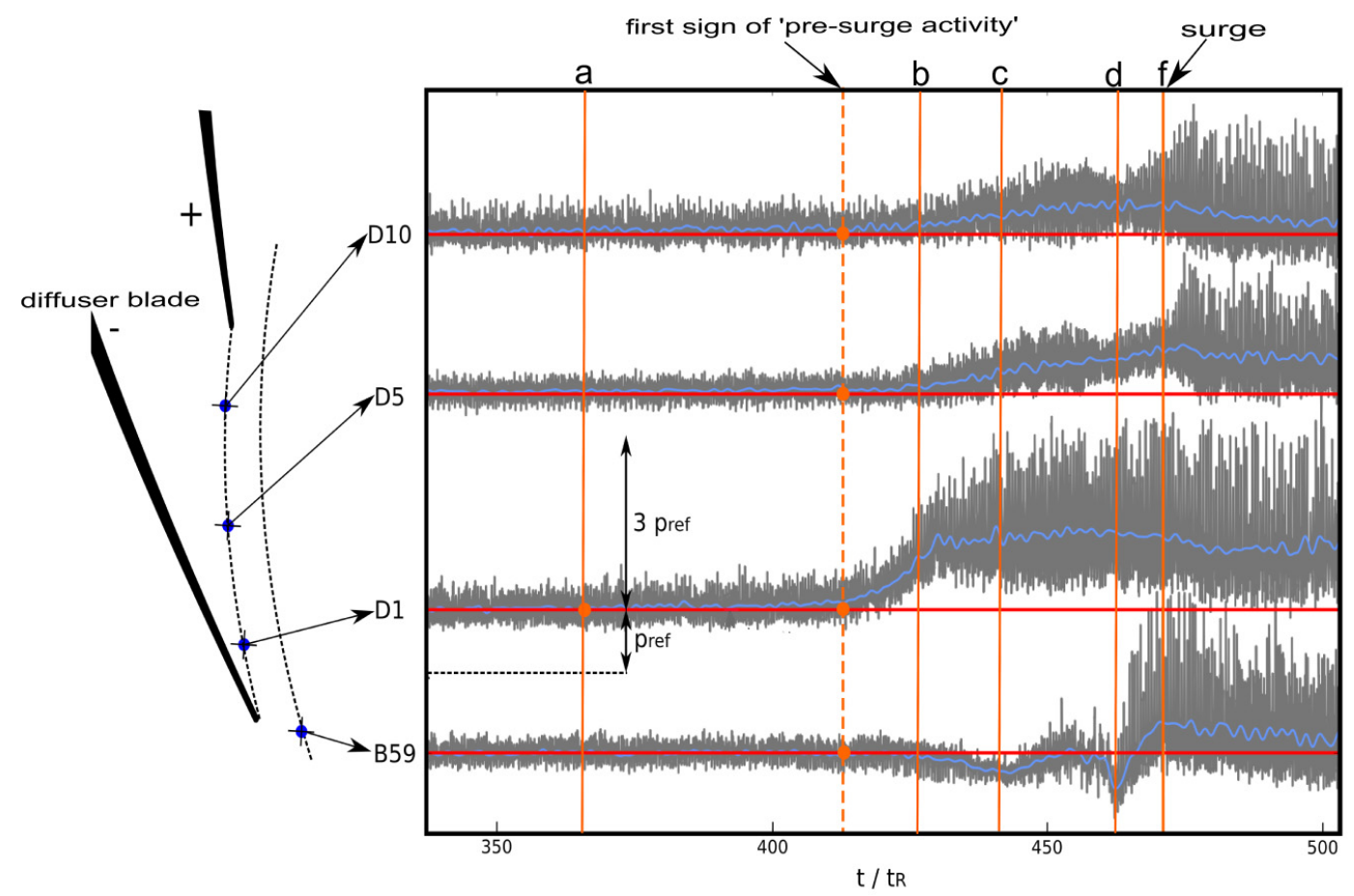

Fig. 7. Pressure signals at sensors B59, D1, D5, D10 (0.927Nn). 

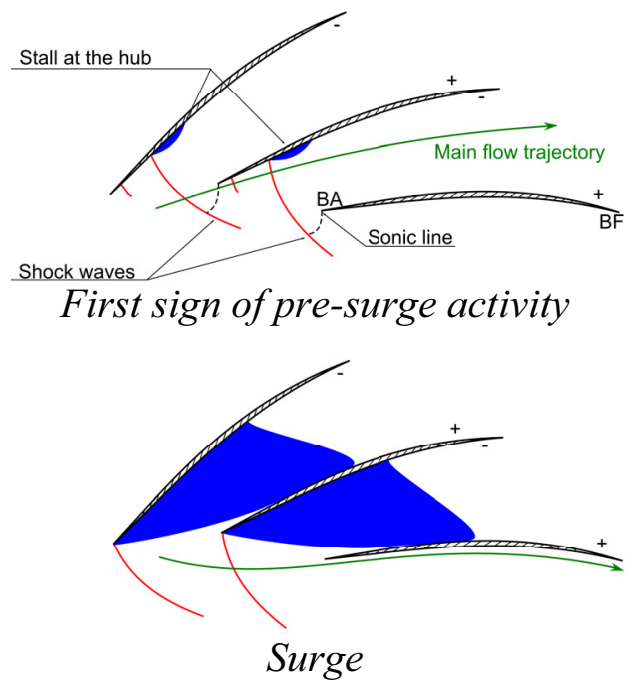

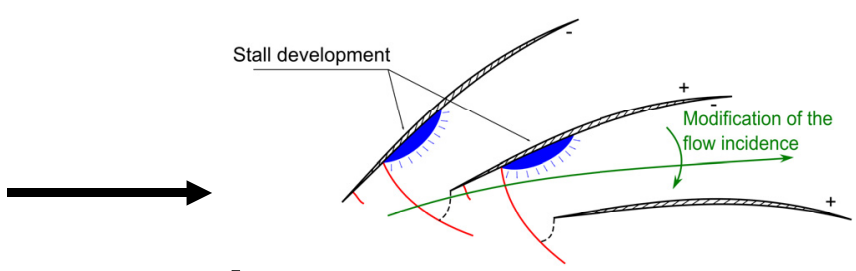

Instant (b)

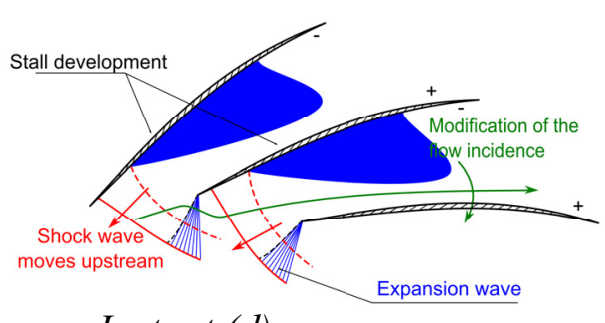

Instant $(d)$

Fig. 8. Scenario of the surge onset at high rotation speeds.
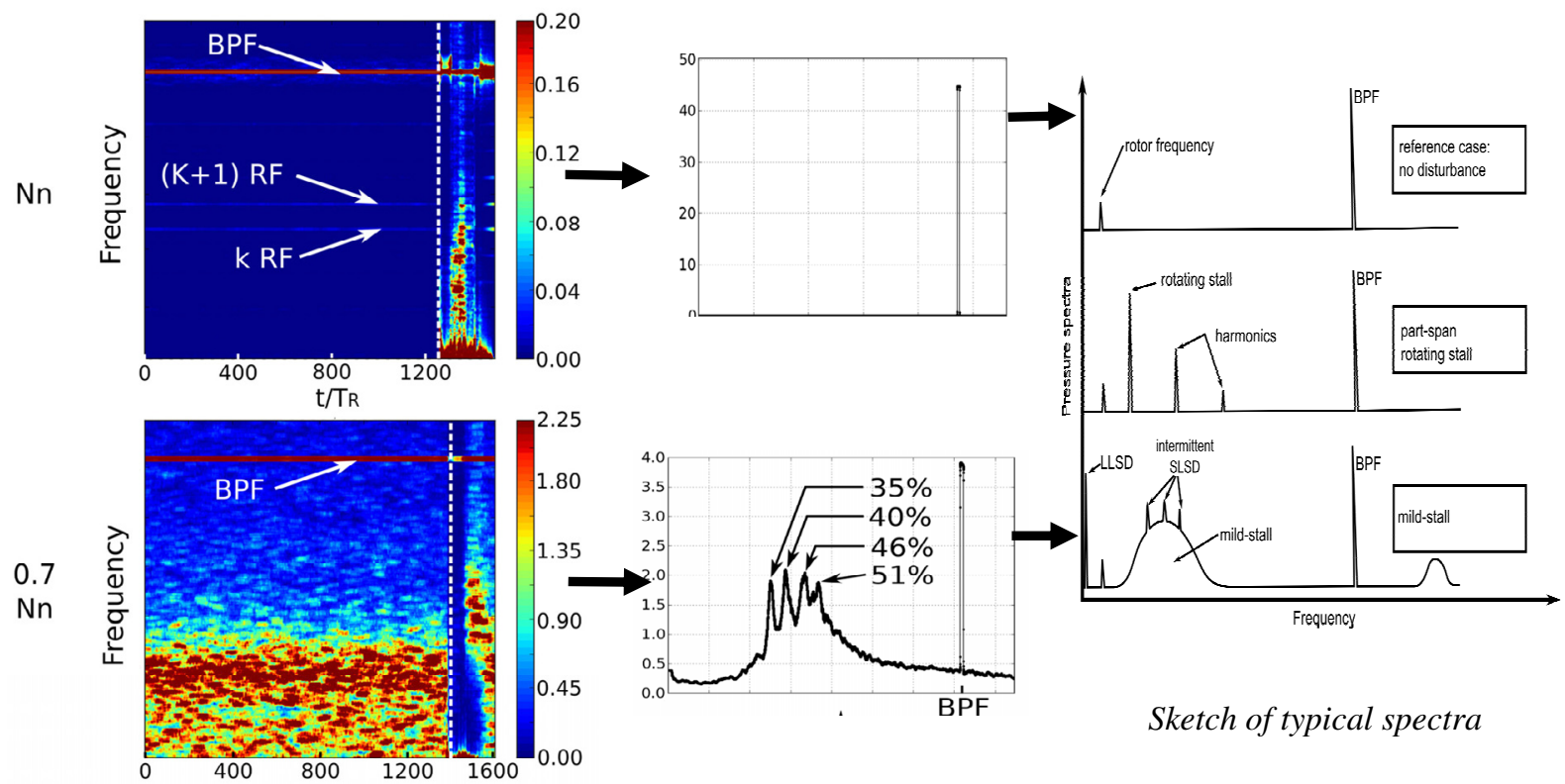

Fig. 9. PSD of pressure fluctuations at sensor I1.

due to the enlargement of the separated bubble. A sketch of the main flow pattern change from the first sign of pre-surge activity to the surge is proposed in Figure 8. The duration between the first pre-surge activity and the surge is around fifty times the blade passing period, it means $5 \times 10^{-3}$ s which excludes the possibility to control the surge inception without controlling the vane suction side flow separation by aspiration for example [13]. Whatever the rotation speed the surge onset remains triggered by the diffuser even if at low speeds the separation results from the combined effects of an over-incidence and the adverse pressure gradient at the throat (and obviously not from a boundary layer-shock wave interaction). Nevertheless pressure fluctuations filtered below the blade passing frequency $\left(\tilde{p}^{B P F}\right)$ are registered in the inducer at lower speeds as discussed below.

\section{Instabilities in the inducer}

Figure 9 gives the power spectral density of the pressure fluctuations (at sensor I1, cf. Fig. 2), reduced by the total energy of the spectrum. The abscissa is the rotor revolutions and the ordinate is the frequency scaled between zero and just beyond the blade passing frequency (BPF). The dotted white line marks the surge. At Nn (top-left) before surge almost all the energy of the spectrum is concentrated in the BPF (a very small amount is due to the Rotor Frequency - RF - and harmonics) whereas at $0.7 \mathrm{Nn}$ (bottom-left) a high activity at low frequency is clearly evidenced. The associated spectra therefore reveal only the BPF at Nn (top-middle) whereas a hump at around forty percent of the BPF is obtained at $0.7 \mathrm{Nn}$ (bottommiddle). Such a hump is characteristic of mild-stall which 
may be understood as a succession of intermittent SLSD (bottom-right). The mild-stall has thus to be differentiated from the rotating stall which is characterized by a spectrum with well-defined frequencies (middle-right). The analysis of the pressure signals registered at all the sensors in the inducer proved that the experienced mildstall results from disturbances (SLSD) emanating from the blade leading edge and due to an over-incidence of the incoming flow at low mass flow rate and low rotation speed (the same behavior is registered at $0.6 \mathrm{Nn}$ ). The disturbances come from cells generated at the leading edge of the impeller which are then transported downstream with a merging process. The displacement of the cells is determined thanks to an analysis of the cross-correlation of the pressure signals at the various sensors. The analysis of the time-lag between all the signals led to the conclusion that the disturbances move along a tip clearance flow trajectory and can therefore be named tip clearance rotating disturbances.

Of course, mild-stall may develop into rotating stall. But in the present case, the surge occurs in the diffuser before instabilities have time to evolve into rotating stall.

\section{Conclusion}

Time-dependent pressure measurements were carried out in a centrifugal compressor stage from 60 to 100 percent of rotation speed. Whatever the rotation speed, the surge is triggered in the diffuser by a massive flow separation on the vane suction side. The time delay between the first pre-surge activity and the surge is only $5 \times 10^{-3} \mathrm{~s}$ which reveals the sudden character of the surge onset. At low rotation speed aerodynamic disturbances were also experienced in the inducer. These disturbances are short length scale disturbances (SLSD) originating in the blade leading edge and moving along the tip leakage trajectory. Their intermittent behaviour leads to mild-stall which cannot develop into rotating stall before the surge occurs in the diffuser.

\section{References}

[1] N.A. Cumpsty, Compressor Aerodynamics, Krieger Publishing Compagny, 2004, ISBN: 1-5724-247-8

[2] E.M. Greitzer, Surge and Rotating Stall in Axial Flow Compressors. Part I: Theoretical Compression System Model, J. Eng. Power 98 (1976) 190-198

[3] E.M. Greitzer, Surge and Rotating Stall in Axial Flow Compressors. Part II: Experimental Results and Comparison With Theory, J. Eng. Power 98 (1976) 199-217

[4] I.J. Day, The Fundamentals of Stall and Surge. Part I: Stall, Von Karman Institute for Fluid Dynamics, Lecture Series 2006-06, Adv. Axial Compressor Aerodynamics (2006) $1-38$

[5] T.R. Camp, I.J. Day, A Study of Spike and Modal Stall Phenomena in a Low-Speed Axial Compressor, J. Turbomach. 120 (1998) 393-401

[6] Z.S. Spakovszky, C.H. Roduner, Spike and Modal Stall Inception in an Advanced Turbocharger Centrifugal Compressor, J. Turbomach. 131 (2009) 031012

[7] R. Van den Braembussche, Stability and Range in Centrifugal Compressors, Von Karman Institute, 1996

[8] R. Van den Braembussche, Surge and Stall in Centrifugal Compressors, Von Karman Institute LS, 1984

[9] N. Bulot, X. Ottavy, I. Trébinjac, Unsteady Pressure Measurements in a High-Speed Centrifugal Compressor, J. Thermal Sci. Science Press 19 (2010) 1-8

[10] J.A. Raw, Surge Margin Enhancement by Porous Throat Diffuser, Can. Aeronautics Space J. 32 (1986) 54-61

[11] D. Japikse, Centrifugal Compressor Design and Performance, Concepts ETI, Inc., 1996

[12] I. Trébinjac, N. Bulot, X. Ottavy, N. Buffaz., Surge inception in a transonic centrifugal compressor stage, paper GT 2011-45116, ASME TurboExpo 2011

[13] A. Marsan, I. Trébinjac, S. Coste, G. Leroy, Study and Control of a Radial Diffuser Stall, Int. J. Rotating Mach. 2012, Article ID 549048, 2012 